\title{
Comparison Between Aerobic Power Parameters at Different Time- Averaging Intervals in Swimming: an Update
}

\author{
Sousa $\mathrm{A}^{1}$, de Jesus $\mathrm{K}^{1}$, Figueiredo $\mathrm{P}^{1,2}$, Sousa $\mathrm{M}^{1}$, Vilas-Boas $\mathrm{JP}^{1,3}$ and Fernandes $\mathrm{RJ}^{1,3, *}$ \\ ${ }^{I}$ Centre of Research, Education, Innovation and Intervention in Sport, Faculty of Sport, University of Porto, Porto, \\ Portugal \\ ${ }^{2}$ Higher Education Institute of Maia, Portugal \\ ${ }^{3}$ Porto Biomechanics Laboratory, University of Porto, Porto, Portugal
}

\begin{abstract}
Sousa et al. (Open Sports Sci J, 3: 22 - 24, 2010) showed that different time averaging intervals lead to distinct $\mathrm{VO}_{2}$ values in a maximal $200 \mathrm{~m}$ front crawl effort, evidencing higher $\mathrm{VO}_{2}$ values for breath-by-breath sampling, and differences between this latter data acquisition and all the other less frequent time intervals studied (5, 10, 15 and $20 \mathrm{~s})$. These are interesting outputs in the field of exercise physiology applied to swimming once: (1) $\mathrm{VO}_{2}$ assessment is conducted in a swimming pool with a portable gas analyser which allowed breath-by-breath measurements, and not in a swimming flume with a Douglas bag technique or mixing chamber analyser, as traditionally occurs, and (2) the comparison between different time-averaging intervals used to remove breath-by-breath fluctuations during exercise periods has remained neglected, in sport in general and swimming in particular. Therefore, in the present study, we investigate the influence that different time averaging intervals have in aerobic power related parameters $\left(\mathrm{VO}_{2 \text { peak }}\right.$ and $\mathrm{VO}_{2 \max }$ ). Ten subjects performed $200 \mathrm{~m}$ front crawl effort at supra-maximal intensities (all-out test) and other ten subjects performed $200 \mathrm{~m}$ front crawl effort at maximal aerobic intensities $\left(100 \%\right.$ of $\left.\mathrm{VO}_{2 \max }\right)$. The intensity at which the $200 \mathrm{~m}$ front crawl was performed (supra-maximal and maximal intensities) had a significant effect on $\mathrm{VO}_{2 \text { peak }}$ and $\mathrm{VO}_{2 \max } \mathrm{values}$ obtained for each averaging intervals studied.
\end{abstract}

Keywords: Swimming, time-average intervals, VO2peak, VO2max.

\section{INTRODUCTION}

The goal of competitive swimming is to obtain the fastest speed of locomotion during a race, being success determined by several influencing factors, particularly the energetic and biomechanical ones. This is possible to infer from the swimming performance equation: $\mathrm{v}=\mathrm{E} *(\mathrm{ept} / \mathrm{D})$, where $\mathrm{v}$ is the swimming velocity, $\mathrm{E}$ represents the energy expenditure, ept is the propulsive mechanic efficiency and D represents the hydrodynamic drag [1]. Among the evaluation of the energetic factors, the assessment of maximal oxygen uptake $\left(\mathrm{VO}_{2 \max }\right)$ is a key point of contemporary research in sport science in general and in "swimming science" in particular [2]. Considered to express the maximal metabolic aerobic performance capability of a subject, the $\mathrm{VO}_{2 \max }$ assessment is crucial for a better understanding of human energetics, and therefore, is related to one of the primary areas of interest in swimming training and performance diagnoses $[2,3]$.

Acknowledging that the evaluation of aerobic performance is very relevant for swimming training

*Address correspondence to this author at the Centre of Research, Education, Innovation and Intervention in Sport, Faculty of Sport, University of Porto, Porto, Portugal; Tel: +351220425273;

Fax: +351225500689; E-mail: ricfer@fade.up.pt purposes, it is important to study the specific $\mathrm{VO}_{2}$ kinetics at different swimming intensities. In fact, the physiology of a maximal performance encompasses distinct neuromuscular processes, intramuscular energy turnover, cardiovascular and respiratory elements, which interconnect differently across different swimming intensities [4]. Furthermore, when studying the $\mathrm{VO}_{2}$ response to a specific effort it is essential to analyze the variability on the $\mathrm{VO}_{2}$ data imposed by the used sampling intervals [5]. In fact, the selection of optimal sampling intervals strategy is fundamental to the validation of the research findings, as well as to the correct training diagnosis and posterior prescription of the intensity of the training series [6]. Myers et al. [7] reported 20\% of variability on the $\mathrm{VO}_{2}$ values due to different chosen data sampling intervals, and that the greatest $\mathrm{VO}_{2 \max }$ values were systematically higher as fewer breaths were included in an average. Midgley et al. [8] evidenced that short time-average intervals appear to be inadequate in reducing the noise in pulmonary $\mathrm{VO}_{2}$, resulting in artificially high $\mathrm{VO}_{2 \max }$ values. Moreover, Hill et al. [9] showed higher peak $\mathrm{VO}_{2}\left(\mathrm{VO}_{2 \text { peak }}\right)$ values at different intensities when based on smaller sampling intervals. These last referred studies [7-9] were conducted in laboratory conditions, not in real swimming situation.

Regarding swimming, only our group $[6,10]$ analyzed the $\mathrm{VO}_{2}$ variability when considering distinct time averaging 


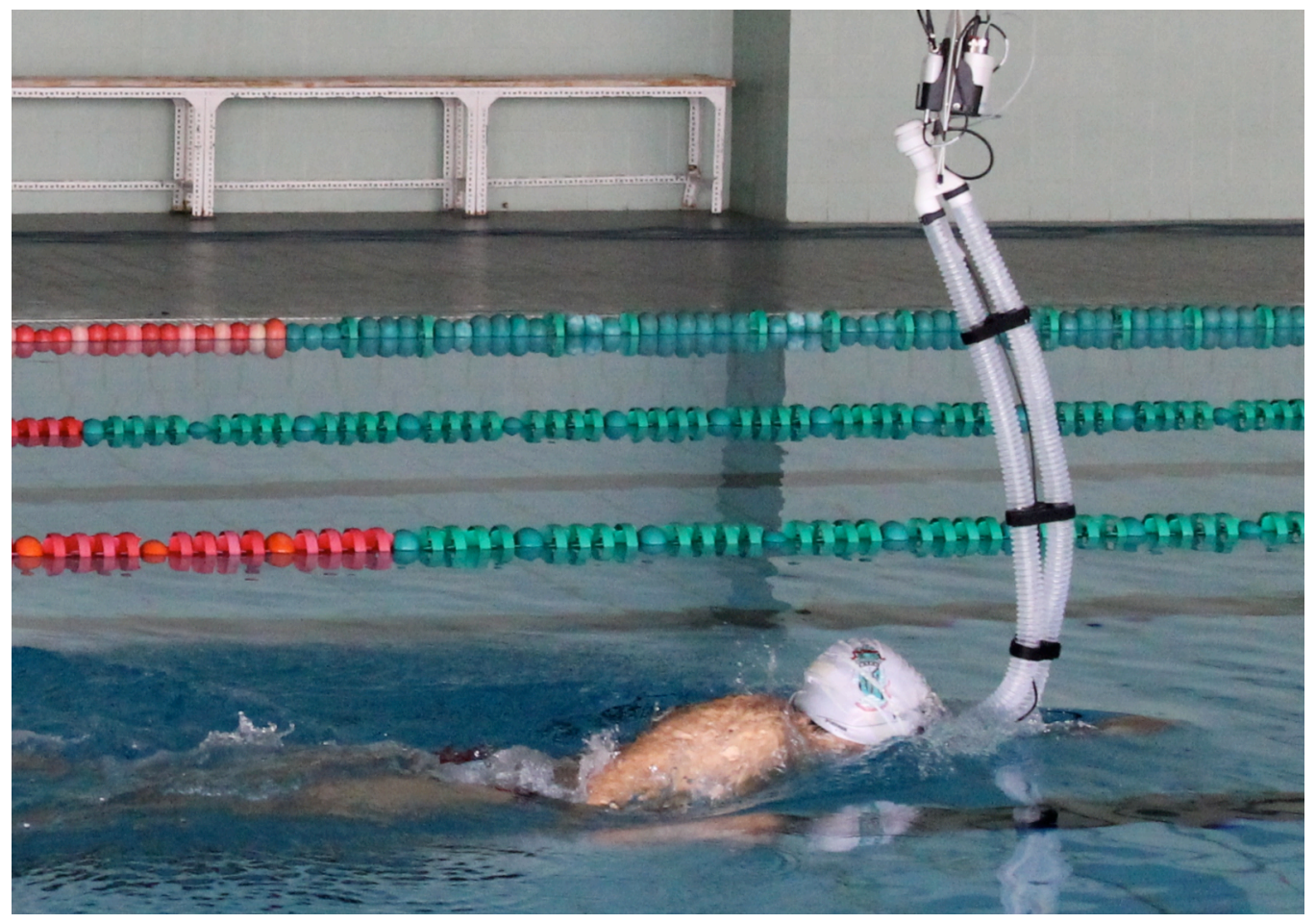

Fig. (1). Specific snorkel and valve system for breath-by-breath $\mathrm{VO}_{2}$ kinetics assessment in swimming.

intervals, but different swimming intensities were never compared. In this sense, the purpose of this study is to compare the variability of the $\mathrm{VO}_{2}$ values obtained in a $200 \mathrm{~m}$ front crawl effort performed at maximal and supramaximal aerobic intensities, using five different time averaging intervals: breath-by-breath and average of 5, 10, 15 , and 20 s, respectively. We hypothesized that the different intensities performed in the $200 \mathrm{~m}$ front crawl would lead to significant effect on $\mathrm{VO}_{2 \text { peak }}$ and $\mathrm{VO}_{2 \max }$ values obtained for each averaging intervals.

\section{METHODS}

\section{Participants}

Ten male well trained swimmers $(20.5 \pm 2.3$ years old, $185.2 \pm 2.3 \mathrm{~cm}, 77.4 \pm 5.3 \mathrm{~kg}$ and $10.1 \pm 1.8 \%$ of fat mass) and ten trained male swimmers $(20.7 \pm 2.8$ years old, $182.0 \pm$ $0.1 \mathrm{~cm}, 75.2 \pm 4.1 \mathrm{~kg}$ and $11.1 \pm 1.6 \%$ of fat mass) volunteered to participate in Sousa et al. [6] and Fernandes et al. [10] studies, respectively. All subjects were informed of the protocol before the beginning the measurement procedures, and were usually involved in physiological evaluation and training control procedures.

\section{Procedures}

Both studies were conducted in a $25 \mathrm{~m}$ indoor swimming pool, $1.90 \mathrm{~m}$ deep, water temperature of $27.5^{\circ} \mathrm{C}$ and humidity of $55 \%$. In Sousa et al., [6], each swimmer performed an allout $200 \mathrm{~m}$ front crawl (with an individual freely chosen pace). $\mathrm{VO}_{2 \text { peak }}$ was accepted as the highest single value on breath-by-breath, $5,10,15$ and 20 s sampling obtained. In Fernandes et al., [10], each swimmer performed a $7 \times 200 \mathrm{~m}$ front crawl intermittent incremental protocol until exhaustion, with $30 \mathrm{~s}$ rest intervals and with velocity increments of $0.05 \mathrm{~m} \cdot \mathrm{s}^{-1}$ between each step. The velocity of the last step was determined through the $400 \mathrm{~m}$ front crawl best time that swimmers were able to accomplish at that moment (using in-water starts and open turns); then, 6 successive $0.05 \mathrm{~m} / \mathrm{s}$ were subtracted from the swimming velocity corresponding to the last step, allowing the determination of the mean target velocity for each step. This was controlled by underwater pacemaker lights (GBK-Pacer, GBK Electronics, Aveiro, Portugal), placed on the bottom of the pool. $\mathrm{VO}_{2}$ data analysis was centred in the step where $\mathrm{VO}_{2 \max }$ occurred, being this considered as the average values of the breath-by-breath, 5, 10, 15 and 20s sampling obtained.

As swimmers were attached to a respiratory valve (cf. Fig. 1), allowing measuring the $\mathrm{VO}_{2}$ kinetics in real time, open turns without underwater gliding and in-water starts were used. For a detailed description of the breathing snorkels used in the supra-maximal and maximal intensities cf. Keskinen et al. [11] and Fernandes and Vilas-Boas [2], respectively. These respiratory snorkels and valve systems were previously considered to produce low hydrodynamic resistance and, therefore, not significantly affect the swimmers performance. $\mathrm{VO}_{2}$ kinetics was measured breathby-breath by a portable metabolic cart ( $\mathrm{K} \mathrm{b}^{2}$, Cosmed, Italy) that was fixed over the water (at a $2 \mathrm{~m}$ height) in a steel cable, allowing following the swimmer along the pool and minimizing disturbances of the swimming movements during the test.

\section{Statistical Analysis}

Mean \pm SD computations for descriptive analysis were obtained for the studied variable using SPSS package (version 14.0 for Windows). In addition, ANOVA of repeated measures was used to test: (i) the differences between the five different sampling intervals considered in the maximal and supra-maximal intensity, and (ii) the interaction effect of intensity in the $\mathrm{VO}_{2}$ values in the five 


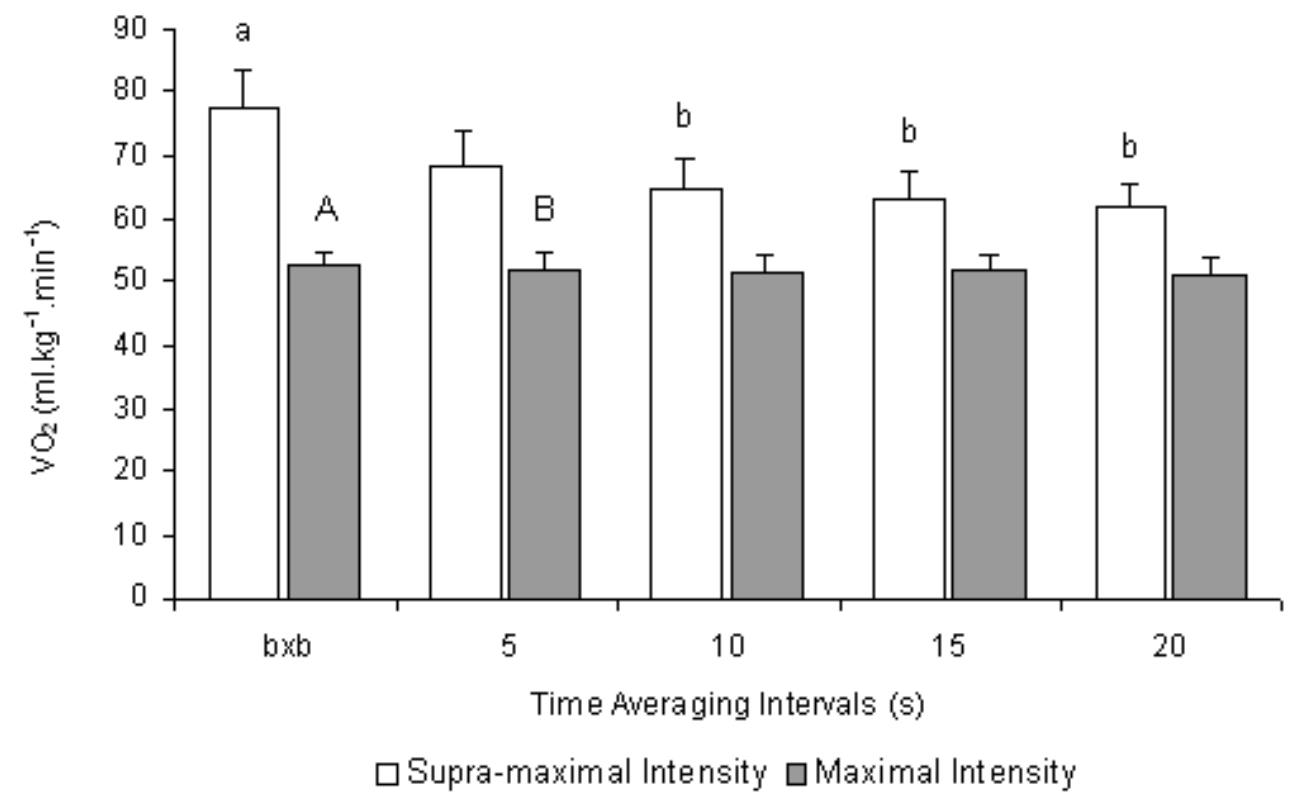

Fig. (2). $\mathrm{VO}_{2}$ values (expressed in $\mathrm{ml} \cdot \mathrm{kg}^{-1} \cdot \mathrm{min}^{-1}$ ) obtained in the breath-by-breath, 5, 10, 15 and 20 s time averaging intervals studied in the $200 \mathrm{~m}$ front crawl effort performed at supra-maximal [10] and maximal aerobic intensities [6]. Bars indicate standard deviations. ${ }^{\text {a }}$ Significantly different from time averaging interval of 5, 10, 15 and $20 \mathrm{~s},{ }^{\mathrm{b}}$ Significantly different from time averaging interval of $5 \mathrm{~s}$, ${ }^{\mathrm{A}}$ Significantly different from time averaging interval of 10,15 and 20 s, respectively, ${ }^{\mathrm{B}}$ Significantly different from time averaging interval of 20s. $P<0.05$.

different sampling intervals studied. When a significant $\mathrm{F}$ value was achieved, Bonferroni post hoc procedures were performed to locate the pairwise differences between the averages. A significance level of 5\% was accepted. Since a limited sample was used, effect size was computed with Cohen's f. It was considered (1) small effect size if $0 \leq|\mathrm{f}| \leq$ 0.10 ; (2) medium effect size if $0.10<|\mathrm{f}| \leq 0.25$; and (3) large effect size if $|\mathrm{f}|>0.25$ [12].

\section{RESULTS}

The $\mathrm{VO}_{2}$ values (expressed in $\mathrm{ml} \cdot \mathrm{kg}^{-1} \cdot \mathrm{min}^{-1}$ ) obtained in the breath-by-breath, 5, 10, 15 and 20s time averaging intervals studied in the $200 \mathrm{~m}$ front crawl effort performed at supra-maximal [10] and maximal aerobic intensities [6] are presented in Fig. (2).

In Sousa et al. [10], $\mathrm{VO}_{2 \text { peak }}$ ranged from 61.1 to 77.7 to $\mathrm{ml} \cdot \mathrm{kg}^{-1} \cdot \mathrm{min}^{-1}\left(F_{(1.82 ; 16.38)}=59.55, P<0.001, f=0.86\right)$. Higher $\mathrm{VO}_{2 \text { peak }}$ values were reported for breath-by-breath interval, being observed differences between the $5 \mathrm{~s}$ averaging interval and the other less frequent data acquisitions considered (10, 15 and 20s). In Fernandes et al. [6], $\mathrm{VO}_{2 \max }$ ranged from 51.1 to $53.2 \mathrm{ml} \cdot \mathrm{kg}^{-1} \cdot \mathrm{min}^{-1}\left(F_{(2.18 ; 19.63)}=4.12, P<0.05, f=0.31\right)$. The breath-by-breath time interval was only significantly different from the three less frequent averaging intervals studied (10, 15 and 20s), being also reported differences between the 5 and 20 s intervals methods. The intensity at which the $200 \mathrm{~m}$ front crawl was performed (supra-maximal and maximal intensities) had a significant effect on $\mathrm{VO}_{2 \text { peak }}$ and $\mathrm{VO}_{2 \max }$ values obtained for each averaging intervals studied $\left(F_{(1.87 ; 33.75)}=44.15, P<\right.$ $0.001, f=0.71)$.

\section{DISCUSSION}

It is well accepted that for modern diagnostics of swimming performance, new more precise and accurate analytical techniques for $\mathrm{VO}_{2}$ kinetics assessment are needed. In fact, after the Douglas bags procedures, $\mathrm{VO}_{2}$ became to be directly assessed using mixing chamber's devices, and only afterwards an upgrade enabled real time breath-by-breath data collection with portable gas measurement systems [13]. Furthermore, this improvement also allowed testing in normal swimming pool conditions, overlapping the standard laboratory conditions that do not perfectly reflect the real-world performances [2, 3, 15]. The $\mathrm{VO}_{2}$ peak mean value obtained in Sousa et al. [10] study was similar to those described in the literature for experienced male competitive swimmers [14, 15], but higher than the $\mathrm{VO}_{2 \max }$ mean value reported by Fernandes et al. [6]. This may be due to the different intensity domain in which both efforts occurred. In fact, the sudden and exponential increase in $\mathrm{VO}_{2}$ that occurs close to the beginning of the effort at intensities above $\mathrm{VO}_{2 \max }$ triggers the attainment of high $\mathrm{VO}_{2}$ values [3]. Moreover, the intensity at which the $200 \mathrm{~m}$ front crawl was performed (supra-maximal and maximal intensities) had a significant effect $(71 \%)$ on $\mathrm{VO}_{2 \text { peak }}$ and $\mathrm{VO}_{2 \max }$ values obtained for each sampling intervals studied.

Regarding the primary aim of the current study, both Sousa et al. [10] and Fernandes et al. [6] studies corroborate the specialized literature conducted in other cyclic sports (namely treadmill running and cycle ergometer), which state that less frequent sampling frequencies underestimate the $\mathrm{VO}_{2}$ values $[7,16,17]$. Regarding the swimming specialized literature, both studies are unique and both reported that the breath-by-breath acquisition presented greater values than sampling intervals of 10,15 and $20 \mathrm{~s}$. This fact seems to be explained by the greater temporal resolution that breath-bybreath sampling offers, allowing a better examination of small changes in high $\mathrm{VO}_{2}$ values. However, it should be taken into account that the breath-by-breath gas acquisition could induce a significant variability of the $\mathrm{VO}_{2}$ values acquired. Moreover, while Sousa et al. [10] evidenced 
significant differences between the two shortest sampling intervals (breath by breath and 5s), Fernandes et al. [6] only reported significant differences between the breath by breath and time sampling interval of 10,15 and 20 s, and between time sampling interval of 5 and 20s. These apparently incongruent results may be due to the distinct swimming intensities at which both efforts occurred.

In conclusion, we have shown that the intensity at which the $200 \mathrm{~m}$ front crawl was performed (supra-maximal and maximal intensities) had a significant effect on $\mathrm{VO}_{2 \text { peak }}$ and $\mathrm{VO}_{2 \max }$ values obtained for each averaging intervals studied, still being unanswered which of the models tested is the most appropriate sampling interval to be used. In this sense, in $\mathrm{VO}_{2 \text { peak }}$ and $\mathrm{VO}_{2 \max }$ assessment it must be taken into account the intensity at which the effort occurred because this may lead to distinct averaging intervals strategies. At supramaximal intensity, and considering the higher ventilation, respiratory frequency and $\mathrm{VO}_{2}$, the possibility of selecting an artifact with lower averaging intervals (e.g. breath-bybreath), is higher. Such fact is clearly stated in the significant difference between $\mathrm{VO}_{2 \text { peak }}$ values obtained (ranging from 61.1 to 77.7 to $\mathrm{ml} \cdot \mathrm{kg}^{-1} \cdot \mathrm{min}^{-1}$ ). At maximal intensities, being this range lower (51.1 to 53.2 to $\mathrm{ml} \cdot \mathrm{kg}^{-1} \cdot \mathrm{min}^{-1}$ ), the associated error is less obvious. A limitation to our study is the fact that the swimmers who performed the $200 \mathrm{~m}$ front crawl at supra-maximal intensity were not the ones that held the $200 \mathrm{~m}$ at maximal intensity. Such lack of uniformity could lead to inter individual differences possible to interfere in the $\mathrm{VO}_{2 \text { peak }}$ and $\mathrm{VO}_{2 \max }$ values obtained. Future research about this topic, also conducted in ecologic swimming conditions, i.e., in swimming-pool (not in laboratory based ergometers and swimming flumes) is needed. Although $\mathrm{VO}_{2}$ is difficult to measure due to technical limitations imposed by the swimming pool and the aquatic environment, its assessment in non-ecological conditions could influence the $\mathrm{VO}_{2 \max }$, compromise the assessment of the corresponding velocity at $\mathrm{VO}_{2 \max }\left(\mathrm{vVO}_{2 \max }\right)$ and the time to exhaustion at $\mathrm{vVO}_{2 \max }$. These two latter problems could induce errors in training intensities prescriptions. In this sense, the most advanced (valid, accurate and reliable) monitoring methods that could be used during actual swimming must be used in order to assess $\mathrm{VO}_{2}$ in ecological swimming conditions, allowing more reliable, accurate and valid results.

The selection of optimal sampling strategies is fundamental to the validation and comparison of research findings, as well as to the correct training diagnosis and training intensities prescription. Literature results should be taken with caution when comparing $\mathrm{VO}_{2 \text { peak }}$ and $\mathrm{VO}_{2 \max }$ values assessed with different sampling intervals and in different intensity domains. In addition, a standardized criterion should be found to accurate set the $\mathrm{VO}_{2 \text { peak }}$ and $\mathrm{VO}_{2 \max }$ that removes the possibility of selecting an artifact.

\section{CONFLICT OF INTEREST}

The authors confirm that this article content has no conflicts of interest.

\section{ACKNOWLEDGEMENTS} 9577).

PTDC/DES/101224/2008 (FCOMP-01-0124-FEDER-00-

\section{REFERENCES}

[1] Pendergast D, Di Prampero E, Craig A, Wilson D, Rennie D. Quantitative analysis of the front crawl in men and women. J Appl Physiol 1977; 43(3): 475-9.

[2] Fernandes RJ, Vilas-Boas JP. Time to exhaustion at the VO2max velocity in swimming: a review. J Hum Kinet 2012; 32: 121-34.

[3] Sousa A, Figueiredo P, Oliveira N, et al. VO2 kinetics in 200-m race-pace front crawl swimming. Int J Sports Med 2011; 32: 1-6.

[4] Aspenes S, Karlsen T. Exercise-Training intervention studies in competitive swimming. Sports Med 2012; 42(6): 527-43.

[5] Dwyer D. A standard method for the determination of maximal aerobic power from breath-by-breath $\mathrm{VO}_{2}$ data obtained during a continuous ramp test on a bicycle ergometer. J Exerc Physiol 2004; $7(5) ; 1-9$.

[6] Fernandes RJ, de Jesus K, Baldari C, et al. Different VO2max time-averaging intervals in swimming. Int $\mathbf{J}$ Sports Med 2012; 33(12): 1010-5.

[7] Myers J, Walsh D, Sullivan M, Froelicher V. Effect of sampling on variability and plateau in oxygen uptake. J Appl Physiol 1990; 68(1): 404-10.

[8] Midgley A, McNaughton L, Carroll S. Effect of the VO2 timeaveraging interval on the reproducibility of VO2max in healthy athletic subjects. Clin Physiol Funct Imaging 2007; 27: 122-5.

[9] Hill DW, Stephens LP, Blumoff -Ross SA, Poole DC, Smith JC. Effect of sampling strategy on measures of VO2 peak obtained using commercial breath-by-breath systems. Eur J Appl Physiol 2003; 89: 564-9.

[10] Sousa A, Figueiredo P, Oliveira N, et al. Comparison between swimming VO2peak and VO2max at different time intervals. Open Sports Sci J 2010; 3: 22-4.

[11] Keskinen K, Rodriguez F, Keskinen O. Respiratory snorkel and valve system for breath-by-breath gas analysis in swimming. Scand J Med Sci Sports 2003; 13: 322-9.

[12] Cohen J. Statistical power analysis for the behavioral sciences. $2^{\text {nd }}$ ed. Hillsdale: Lawrence Erlbaum Associates 1988.

[13] Fernandes RJ, Figueiredo P, Vilas-Boas JP. About the use and conclusions extracted from a single tube snorkel for respiratory data acquisition during swimming. J Physiol Sci 2013; 63(2): 1557.

[14] Rodriguez F, Mader A. Energy metabolism during 400 and 100m crawl swimming: Computer simulation based on free swimming measurements. In: Chatard JC, Ed. Proceedings of the IXth World Symposium on Biomechanics and Medicine in Swimming; SaintÉtienne: Publications de l'Université de Saint-Étienne; 2003; pp. 373-8.

[15] Fernandes RJ, Keskinen K, Colaço P, et al. Time limit at VO2max velocity in elite crawl swimmers. Int J Sports Med 2008; 29: 14550 .

[16] Astorino A, Robergs R. Influence of time-averaging on the change in VO2 at VO2max. Med Sci Sports Exerc 2001; Suppl 1: 33(5):S45.

[17] Astorino A. Alterations in $\mathrm{VO}_{2} \max$ and the $\mathrm{VO}_{2}$ plateau with manipulation of sampling interval. Clin Physiol Funct Imaging 2009; 29: 60-7. 